\title{
Prediction Model of Lymph Node Metastasis Risk in Elderly Patients with Early Gastric Cancer before Endoscopic Resection: A Retrospective Analysis Based on International Multicenter Data
}

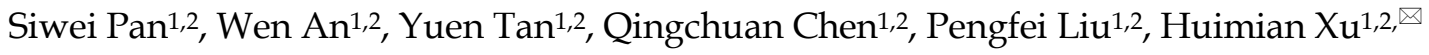 \\ 1. Department of Surgical Oncology, The First Affiliated Hospital of China Medical University, Shenyang 110001, Liaoning, China \\ 2. Department of Surgical Oncology and General Surgery, Key Laboratory of Precision Diagnosis and Treatment of Gastrointestinal Tumors, Ministry of \\ Education, The First Affiliated Hospital of China Medical University, Shenyang, China \\ $\triangle$ Corresponding author: Huimian Xu, MD, PhD. Mailing address: Department of Surgical Oncology, the First Affiliated Hospital of China Medical University, \\ North Nanjing Street 155, Shenyang 110001, China. E-mail: xuhuimian@126.com; Phone: +86-24-83283556; Fax: +86-24-83283556 \\ (1) The author(s). This is an open access article distributed under the terms of the Creative Commons Attribution License (https://creativecommons.org/licenses/by/4.0/). \\ See http://ivyspring.com/terms for full terms and conditions.
}

Received: 2020.12.02; Accepted: 2021.07.09; Published: 2021.07.25

\begin{abstract}
Background: Patients with early gastric cancer (EGC) must suffer reoperation if diagnosed with a high possibility of lymph node (LN) metastasis. The purpose of the current study was to develop and validate a model to predict the risk of $\mathrm{LN}$ metastasis in elderly patients before endoscopic resection.

Methods: A total of 1911 EGC patients who had undergone radical surgery were selected and assigned randomly $(2: 1)$ to either the training cohort or the validation cohort. A nomogram was established based on the univariate and multivariate logistic regression models using the training cohort. Cox proportional hazards regression models were applied to identify the prognostic factors in univariate and multivariable analyses.

Results: Three variables-tumor size, grade, and T stage - were derived from the multivariate analyses in the training cohort and incorporated into the nomogram. The AUC of the nomogram was 0.732 in the training cohort and 0.706 in the validation cohort. There were significant differences in survival among patients with different degrees of $\mathrm{LN}$ metastasis risk (training cohort: five-year disease-specific survival (DSS): low risk $88.1 \%$ vs. moderate risk $80.0 \%$ vs. high risk $72.9 \%, P<0.001$; validation cohort five-year DSS: low risk $89.0 \%$ vs. moderate risk $84.3 \%$ vs. high risk $72.2 \%, \mathrm{P}<0.001$ ). The $\mathrm{LN}$ metastasis risk assessed from the model was also an independent prognostic factor.

Conclusion: We established a nomogram that accurately predicts $L N$ metastasis risk for elderly patients with EGC before endoscopic resection to avoid further injury from reoperation.
\end{abstract}

Key words: Early gastric cancer, lymph node metastasis, elderly patients, nomogram

\section{Introduction}

Early gastric cancer (EGC) in which tumor invasion is restricted to the mucosa (T1a) or submucosa (T1b), regardless of lymph node (LN) metastasis status, has a significantly more favorable prognosis than advanced gastric cancer (GC), and has an outstanding five-year survival rate following radical resection $[1,2]$. Currently, endoscopic submucosal dissection (ESD) and endoscopic mucosal resection (EMR) have been recognized as standard treatments that can achieve en bloc resection of EGC $[3,4]$. However, the above treatments do not effectively dissect perigastric LNs. Patients could also require additional radical gastrectomy after diagnosis of high-risk LN metastasis following postoperative pathological examination [5-7]. The LN metastasis rate has been reported in previous studies to range from $8.5 \%$ to $20.1 \%$ [8-10].

Since LN metastasis is recognized as an 
independent prognostic factor for GC, the occurrence of LN metastasis will greatly increase the risk of postoperative recurrence and shorten postoperative survival time in EGC $[2,11,12]$. It was reported that EGC patients with metastasis LNs (mLNs) had more than five times the risk of postoperative recurrence than EGC patients without metastasis [11]. Therefore, it is of great significance to evaluate the risk of $\mathrm{LN}$ metastasis in advance of treatment decisions.

To date, several studies have developed different prediction models. The eCura system proposed by the Japanese Gastric Cancer Association (JGCA) is more authoritative and is included in the fifth edition of the Japanese Gastric Cancer Treatment Guideline [13, 14]. It evaluates LN metastasis risk of post-ESD patients based on five factors: lymphatic invasion, tumor size, vertical margin, venous invasion, and degree of submucosal invasion. Further, it suggests that patients with intermediate and high risk undergo additional radical gastrectomy. Nevertheless, the shortcoming of this system is that it is evaluated after ESD. Additional surgery might be a severe blow to a patient's body, especially for patients at an advanced age. Thus, a preoperative risk assessment model that can rapidly analyze LN metastasis risk based on clinical data is particularly important for elderly patients.

Hence, in the current study, we aimed to construct a preoperative LN risk assessment model for elderly patients with EGC. We used the First Affiliated Hospital of China Medical University (CMU) and the Surveillance, Epidemiology, and End Results (SEER) databases to identify which group was suitable for direct radical gastrectomy.

\section{Materials and Methods}

\section{Patient Source}

The SEER program has collected and published incidence and survival data based on cancer registries, and covers approximately $26 \%$ of the US population $[15,16]$. The cohort for the current study was selected from the SEER database using SEER-stat software (SEER ${ }^{*}$ Stat 8.3.6). We obtained permission to access research data files with the reference number 10944-Nov2019.

Patients who had undergone radical gastrectomy at the Department of Surgical Oncology, the First Hospital of CMU (Shenyang, China) from January 1980 to December 2012, and patients who had undergone gastrectomy and were subsequently diagnosed with gastric adenocarcinoma between 2000 and 2016 in the SEER database were considered for this study. Patients with GC invading the mucosa (T1a) or submucosa (T1b) were selected. However, patients with tumors located at the cardia or esophagogastric junction were excluded. Patients were also excluded based on the following criteria: (1) younger than 60 or older than 90 years old; (2) the clinical or follow-up information was not clear; (3) the survival time was less than one month; (4) death was from diseases other than GC. After applying these criteria, 1911 patients were included for further analyses.

Sex and age of the patient, size and site of the primary tumor, grade, extent of invasion, number of retrieved and metastatic LNs, adjuvant therapy, follow-up duration, and survival status at the last follow-up (SEER cohort: Nov 2018; CMU cohort: Nov 2016) were selected for the current analyses. Tumor, node, and metastasis (TNM) stage was classified according to the eighth edition of the American Joint Committee on Cancer (AJCC) Cancer Staging Manual $[1,17]$.

\section{Development and Validation of the Nomogram}

After the random distribution, two-thirds of the patients older than 60 years were assigned to the training cohort $(\mathrm{n}=1274)$ and the rest were assigned to the validation cohort $(n=637)$.

To identify the specific predictors for LN metastasis, a logistic regression model was used to evaluate the correlation between the occurrence of LN metastasis and the following factors in univariate and multivariable analyses: sex, age, primary site and size of the tumor, grade, and $\mathrm{T}$ stage (which could be obtained in preoperative examinations). Afterward, based on the logistic regression model results, a nomogram was constructed to assess the risk of LN metastasis. Adjuvant therapy, both preoperatively and postoperatively, is performed with slightly different protocols and screening in different areas, which may affect the effectiveness of the construction and validation of prediction model, especially the current preoperative prediction model. Balachandran et al. also indicated that treatment should be avoided as a covariate in the prediction model unless there were validated data from a randomized clinical trial [18]. In the light of these considerations, we did not include neoadjuvant therapy in our prediction model.

The performance of the nomogram was evaluated from the perspective of discrimination and calibration. Discrimination was evaluated by the receiver operating characteristic (ROC) curve and area under the curve (AUC), which reflect the sensitivity, specificity, and accuracy of the model $[19,20]$. Calibration curves were performed by comparing the predicted risk of LN metastasis from the nomogram with the observed actual incidence [21]. We used the 
bootstrapping (1000 repetitions) method to reduce the bias. An established nomogram was used to calculate the score for each of the patient validation groups for external nomogram validation. Finally, to measure clinical utility, a decision curve analysis (DCA) was conducted by measuring the net benefits for a group of threshold probabilities.

\section{Statistical Analysis}

Disease-specific survival (DSS) was defined as the survival time from resection to death due to GC. The Kaplan-Meier method was applied to calculate DSS and was verified by the log-rank test. The categorical variables were described as counts and proportions. Cox proportional hazards regression models were applied to identify the prognostic factors in univariate and multivariable analyses. The Kruskal-Wallis test was used to confirm the relationship between $\mathrm{pN}$ stage and $\mathrm{LN}$ metastasis risk groups. The reverse Kaplan-Meier method was used to quantify follow-up [22-24].

Statistical analyses were carried out using $R$ software (version 3.5.3; R Foundation for Statistical Computing, Vienna, Austria) and SPSS (version 23.0; SPSS Inc., Chicago, IL). A two-tailed P-value < 0.05 was considered statistically significant in all analyses.

\section{Results}

\section{Clinicopathological Characteristics and Survival Analyses}

A total of 1911 EGC patients over 60 years old were found for further study, including 1763 from the SEER database and 148 from the CMU database. The demographic and pathological characteristics are illustrated in Table 1. More than half of the patients were male. The median ages of the CMU cohort and the SEER cohort were 69 and 73 years, respectively. The median follow-up times calculated by the reverse Kaplan-Meier method were 103 months for the CMU cohort and 66 months for the SEER cohort. Moreover, the incidence of LN metastasis was $21.6 \%$ in the CMU cohort and $19.2 \%$ in the SEER cohort. More than 50\% of LN metastatic patients had one or two mLNs (N1 stage). Of the patients without mLNs in both cohorts, the distribution of patients with T1a tumors and patients with $\mathrm{T} 1 \mathrm{~b}$ tumors was similar. However, over $80 \%$ (313 of 371$)$ of patients with mLNs were diagnosed with $\mathrm{T} 1 \mathrm{~b}$ tumors.

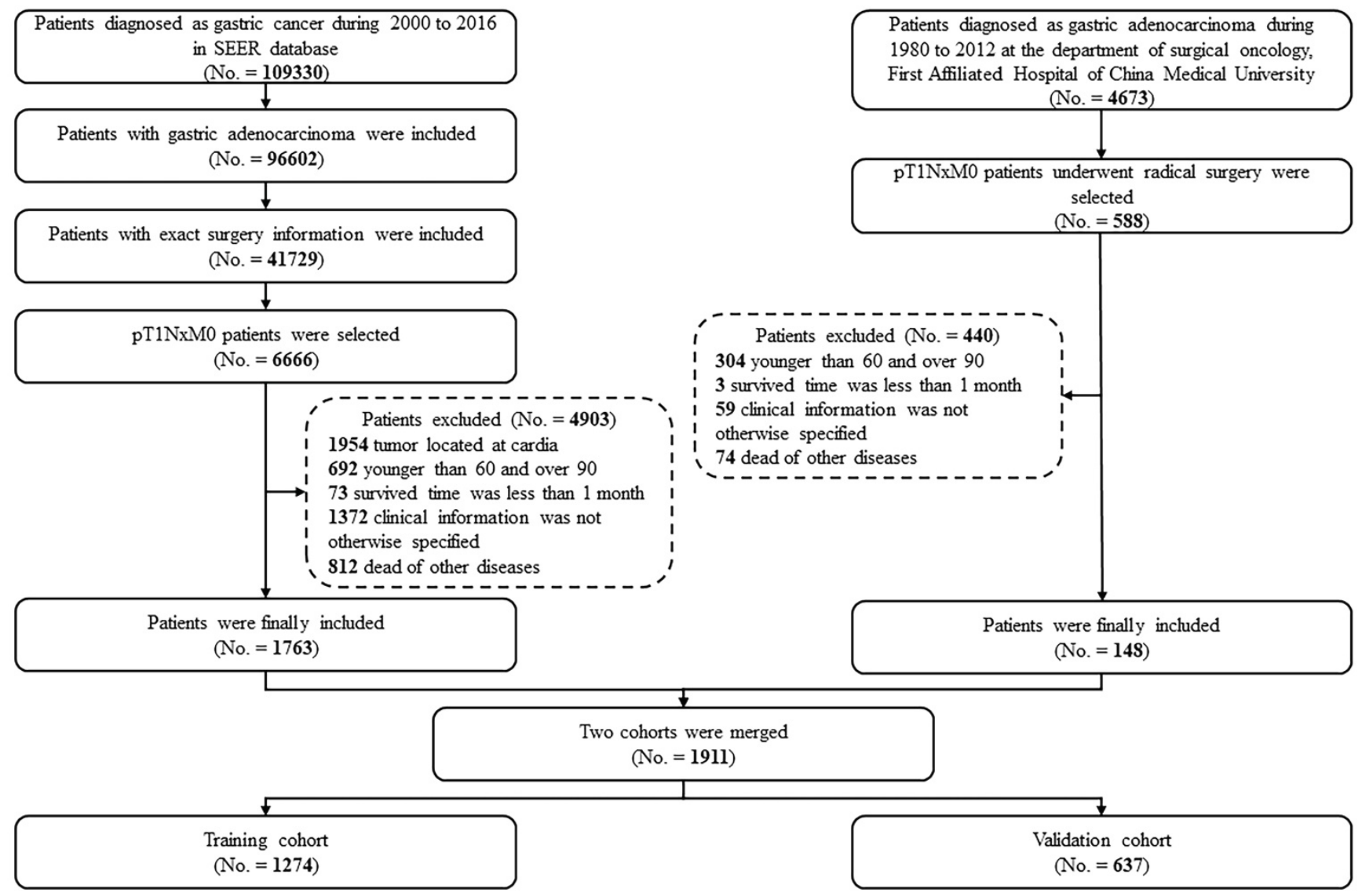

Figure 1. Case screening process for the current analyses from the CMU and SEER database. 
(A)

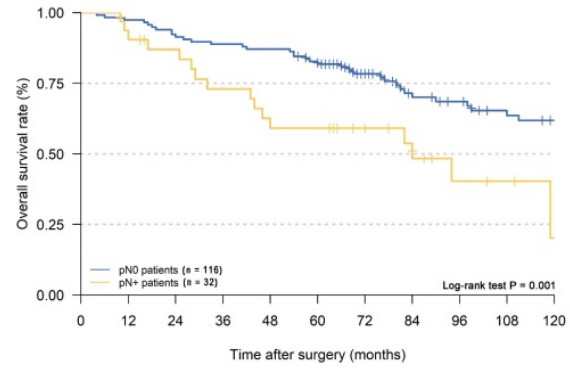

(B)

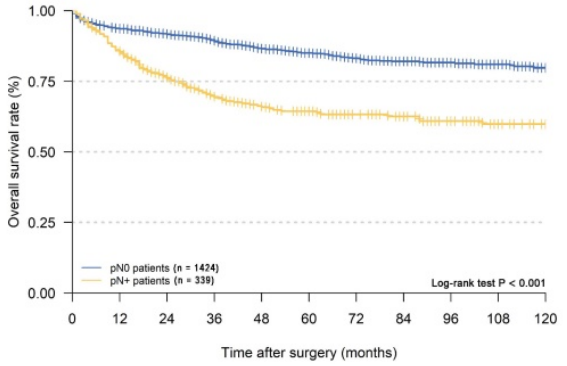

(C)

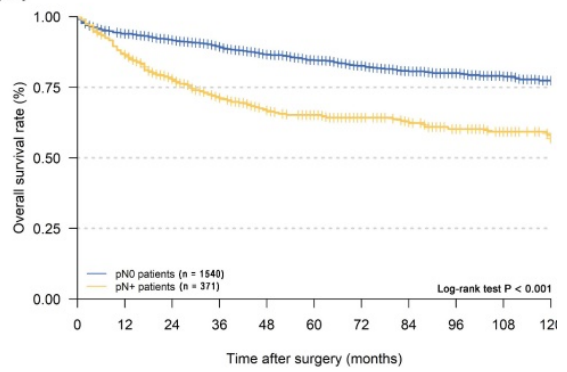

Figure 2. Kaplan-Meier disease-specific survival curves of elderly EGC patients with or without lymph node metastasis: (A) CMU cohort; (B) SEER cohort; (C) merged cohort.

Table 1. Demographic and pathological characteristics of the 1911 patients in the current study.

\begin{tabular}{|c|c|c|c|c|c|c|}
\hline \multirow[t]{2}{*}{ Characteristics } & \multicolumn{3}{|c|}{$\mathrm{CMU}$ cohort $($ No. $=148)$} & \multicolumn{3}{|c|}{ SEER cohort $($ No. $=1763)$} \\
\hline & All & $\begin{array}{l}\text { N0 (No. }= \\
116,78.4 \%)\end{array}$ & $\begin{array}{l}\mathrm{N}+(\text { No. }= \\
32,21.6 \%)\end{array}$ & All & $\begin{array}{l}\text { N0 (No. }= \\
1424,80.8 \%)\end{array}$ & $\begin{array}{l}\mathrm{N}+(\text { No. }= \\
339,19.2 \%)\end{array}$ \\
\hline \multicolumn{7}{|l|}{ Sex } \\
\hline Male & 116 & $92(79.3 \%)$ & $24(75.0 \%)$ & 960 & $770(54.1 \%)$ & $190(56.0 \%)$ \\
\hline Female & 32 & $24(20.7 \%)$ & $8(25.0 \%)$ & 803 & $654(45.9 \%)$ & $149(44.0 \%)$ \\
\hline \multicolumn{7}{|l|}{ Tumor location } \\
\hline Upper & 10 & $10(8.6 \%)$ & $0(0.0 \%)$ & 61 & $51(3.6 \%)$ & $10(2.9 \%)$ \\
\hline Middle & 20 & $14(12.1 \%)$ & $6(18.8 \%)$ & 281 & $233(16.4 \%)$ & $48(14.2 \%)$ \\
\hline Lower & 99 & $78(67.2 \%)$ & $21(65.6 \%)$ & 808 & $641(45.0 \%)$ & $167(49.3 \%)$ \\
\hline Mixed & 19 & $14(12.1 \%)$ & $5(15.6 \%)$ & 613 & $499(35.0 \%)$ & $114(33.6 \%)$ \\
\hline \multicolumn{7}{|l|}{ Grade } \\
\hline Well differentiated & 40 & $28(24.1 \%)$ & $12(37.5 \%)$ & 264 & $245(17.2 \%)$ & $19(5.6 \%)$ \\
\hline $\begin{array}{l}\text { Moderately } \\
\text { differentiated }\end{array}$ & 29 & $20(17.2 \%)$ & $9(28.1 \%)$ & 622 & $512(36.0 \%)$ & $110(32.4 \%)$ \\
\hline $\begin{array}{l}\text { Poorly } \\
\text { differentiated }\end{array}$ & 75 & $65(56.0 \%)$ & $10(31.3 \%)$ & 845 & $645(45.3 \%)$ & $200(59.0 \%)$ \\
\hline Undifferentiated & 4 & $3(2.7 \%)$ & $1(3.1 \%)$ & 32 & $22(1.5 \%)$ & $10(3.0 \%)$ \\
\hline \multicolumn{7}{|l|}{ Size } \\
\hline$\leq 1 \mathrm{~cm}$ & 16 & $16(13.8 \%)$ & $0(0.0 \%)$ & 399 & $367(25.8 \%)$ & $32(9.4 \%)$ \\
\hline$>1 \mathrm{~cm}$ and $\leq 2 \mathrm{~cm}$ & 52 & $43(37.1 \%)$ & $9(28.1 \%)$ & 517 & $441(31.0 \%)$ & $76(22.4 \%)$ \\
\hline$>2 \mathrm{~cm}$ and $\leq 3 \mathrm{~cm}$ & 34 & $26(22.4 \%)$ & $9(28.1 \%)$ & 359 & $280(19.7 \%)$ & $79(23.3 \%)$ \\
\hline$>3 \mathrm{~cm}$ and $\leq 4 \mathrm{~cm}$ & 25 & $17(14.7 \%)$ & $8(25.0 \%)$ & 214 & $159(11.2 \%)$ & $55(16.3 \%)$ \\
\hline$>4 \mathrm{~cm}$ & 20 & $14(12.1 \%)$ & $6(18.8 \%)$ & 274 & $177(12.3 \%)$ & $97(28.6 \%)$ \\
\hline \multicolumn{7}{|l|}{ T stage } \\
\hline $\mathrm{T} 1 \mathrm{a}$ & 65 & $60(51.7 \%)$ & $5(15.6 \%)$ & 645 & $592(41.6 \%)$ & $53(15.6 \%)$ \\
\hline $\mathrm{T} 1 \mathrm{~b}$ & 83 & $56(48.3 \%)$ & $27(84.4 \%)$ & 1118 & $832(58.4 \%)$ & $286(84.4 \%)$ \\
\hline \multicolumn{7}{|l|}{ N stage } \\
\hline No & 116 & $116(100 \%)$ & $0(0.0 \%)$ & 1424 & $1424(100 \%)$ & $0(0.0 \%)$ \\
\hline N1 & 19 & $0(0.0 \%)$ & $19(59.4 \%)$ & 208 & $0(0.0 \%)$ & $208(61.4 \%)$ \\
\hline N2 & 9 & $0(0.0 \%)$ & $9(28.1 \%)$ & 98 & $0(0.0 \%)$ & $98(28.9 \%)$ \\
\hline N3a & 4 & $0(0.0 \%)$ & $4(12.5 \%)$ & 25 & $0(0.0 \%)$ & $25(7.4 \%)$ \\
\hline $\mathrm{N} 3 \mathrm{~b}$ & 0 & $0(0.0 \%)$ & $0(0 \%)$ & 8 & $0(0.0 \%)$ & $8(2.3 \%)$ \\
\hline \multicolumn{7}{|l|}{ Examined LNs } \\
\hline$\leq 15$ & 61 & $52(44.8 \%)$ & $9(28.1 \%)$ & 1043 & $881(61.9 \%)$ & $162(47.8 \%)$ \\
\hline$>15$ & 87 & $64(55.2 \%)$ & $23(71.9 \%)$ & 720 & $543(38.1 \%)$ & $177(52.2 \%)$ \\
\hline \multicolumn{7}{|l|}{ Adjuvant therapy } \\
\hline No/ unknown & 139 & $114(98.3 \%)$ & $25(78.1 \%)$ & 1514 & 1359 (95.4\%) & $155(45.7 \%)$ \\
\hline Yes & 9 & $2(1.7 \%)$ & $7(21.9 \%)$ & 249 & $65(4.6 \%)$ & $184(54.3 \%)$ \\
\hline
\end{tabular}

In the survival analyses, we found that EGC patients with mLNs had a significantly poorer prognosis in the merged cohort (Figure 2A, five-year DSS: $84.6 \%$ vs. $65.2 \%, \mathrm{P}<0.001)$. The same outcomes were confirmed in the SEER cohort and the CMU cohort (Figure 2B and 2C, $\mathrm{P}<0.001$ and $\mathrm{P}=0.001$, respectively).

\section{Univariate and Multivariate Analyses for Predictive Factors}

The merged cohort was divided into a training cohort and a validation cohort by a ratio of 2:1. The characteristics are illustrated in Table 2 . The training cohort included 1274 patients and was applied to develop predictive factors and construct a nomogram. The validation cohort included 637 patients and was used to validate the capacity of the nomogram.

Table 2. Demographic and pathological characteristics of the training cohort and validation cohort in the current study.

\begin{tabular}{|c|c|c|c|c|}
\hline \multirow[t]{2}{*}{ Characteristics } & \multicolumn{2}{|c|}{ Training cohort $($ No. $=1274)$} & \multicolumn{2}{|c|}{$\begin{array}{l}\text { Validation cohort }(\text { No. }= \\
637 \text { ) }\end{array}$} \\
\hline & $\begin{array}{l}\text { N0 (No. }= \\
1028)\end{array}$ & $\begin{array}{l}\mathrm{N}+(\text { No. }= \\
246)\end{array}$ & $\begin{array}{l}\text { N0 (No. }= \\
512)\end{array}$ & $\begin{array}{l}\mathrm{N}+(\text { No. }= \\
125)\end{array}$ \\
\hline \multicolumn{5}{|l|}{ Sex } \\
\hline Male & 574 & 140 & 288 & 74 \\
\hline Female & 454 & 106 & 224 & 51 \\
\hline \multicolumn{5}{|l|}{ Tumor location } \\
\hline Upper & 38 & 6 & 23 & 4 \\
\hline Middle & 159 & 39 & 88 & 15 \\
\hline Lower & 482 & 126 & 237 & 62 \\
\hline Mixed & 349 & 75 & 164 & 44 \\
\hline \multicolumn{5}{|l|}{ Grade } \\
\hline Well differentiated & 192 & 25 & 81 & 6 \\
\hline $\begin{array}{l}\text { Moderately } \\
\text { differentiated }\end{array}$ & 351 & 79 & 181 & 40 \\
\hline Poorly differentiated & 465 & 133 & 245 & 77 \\
\hline Undifferentiated & 20 & 9 & 5 & 2 \\
\hline \multicolumn{5}{|l|}{ Size } \\
\hline$\leq 1 \mathrm{~cm}$ & 256 & 20 & 127 & 12 \\
\hline$>1 \mathrm{~cm}$ and $\leq 2 \mathrm{~cm}$ & 328 & 57 & 156 & 28 \\
\hline$>2 \mathrm{~cm}$ and $\leq 3 \mathrm{~cm}$ & 203 & 55 & 103 & 33 \\
\hline$>3 \mathrm{~cm}$ and $\leq 4 \mathrm{~cm}$ & 106 & 46 & 70 & 17 \\
\hline$>4 \mathrm{~cm}$ & 135 & 68 & 56 & 35 \\
\hline \multicolumn{5}{|l|}{ T stage } \\
\hline T1a & 433 & 36 & 219 & 22 \\
\hline $\mathrm{T} 1 \mathrm{~b}$ & 595 & 210 & 293 & 103 \\
\hline \multicolumn{5}{|l|}{ N stage } \\
\hline No & 1028 & 0 & 512 & 0 \\
\hline N1 & 0 & 144 & 0 & 83 \\
\hline N2 & 0 & 76 & 0 & 31 \\
\hline N3a & 0 & 23 & 0 & 6 \\
\hline $\mathrm{N} 3 \mathrm{~b}$ & 0 & 3 & 0 & 5 \\
\hline \multicolumn{5}{|l|}{ Examined LNs } \\
\hline$\leq 15$ & 626 & 107 & 307 & 64 \\
\hline$>15$ & 402 & 139 & 205 & 61 \\
\hline \multicolumn{5}{|l|}{ Adjuvant therapy } \\
\hline No/ unknown & 979 & 122 & 494 & 58 \\
\hline Yes & 49 & 124 & 18 & 67 \\
\hline
\end{tabular}


The univariate and multivariate analyses by the logistic regression model of the training cohort are illustrated in Table 3. The size of the primary tumor, the grade, and the $\mathrm{T}$ stage were identified as factors being significantly associated with the occurrence of LN metastasis (all $\mathrm{P}<0.05$ ). The significant factors were included in multivariate logistic regression analyses, and all maintained significance (all $\mathrm{P}<0.05$ ). Tumors $>4 \mathrm{~cm}$ [odds ratio $(\mathrm{OR})=4.607,95 \%$ confidence interval (CI): 2.644-8.026] had a significantly higher risk of LN metastasis than smaller tumors. A poorer degree of differentiation also reflected a higher occurrence rate of $\mathrm{LN}$ metastasis for EGC patients $(\mathrm{P}=0.014)$. In addition, the risk of $\mathrm{LN}$ metastasis in pT1b patients was approximately 3.419 times higher than pT1a patients $(\mathrm{OR}=3.419,95 \% \mathrm{CI}$ : 2.329-5.019). Furthermore, the size of the primary tumor, the grade, and the $\mathrm{T}$ stage were selected to establish the nomogram to predict the risk of LN metastasis.

\section{Nomogram Development and Validation}

Based on the results of the multivariate analysis, we established a nomogram that could predict the risk of LN metastasis in the training cohort (Figure 3). With this nomogram model, it was easy to obtain an individualized summing score based on selected variables for each patient and estimate the risk of LN metastasis. The AUC in the training cohort for the nomogram to predict LN metastasis was 0.723 (Figure 4A, 95\% CI: 0.692-0.755). Discrimination was also validated in the validation cohort and the AUC was 0.706 (Figure 4B, 95\% CI: 0.658-0.755). To validate similarities between LN metastasis risk predicted by the nomogram model and actual rates, calibration curves were created in both the training and validation cohorts (Figure 4C, 4D). DCA curves also showed that the nomogram for prognostic prediction had a good ability in clinical utility (Figure 4E and 4F). The results illustrated that the nomogram performed well in predicting LN metastasis risk without significant error in either cohort.

\section{Survival and Relationship Analyses of Different Risk Levels of LN Metastasis}

After each patient's individualized summing score was obtained through the nomogram, we divided the patients into three equal groups according to their scores: low-risk group (with a risk of $\mathrm{LN}$ metastasis lower than $11.7 \%$ ), moderate-risk group (with a risk of LN metastasis from $11.7 \%$ to $24.8 \%$ ), and high-risk group (with a risk of LN metastasis over $24.8 \%$ ). The Kaplan-Meier curves of the three groups in both the training and validation cohorts are illustrated in Figures 5A and 5B (training cohort: five-year DSS: low risk $88.1 \%$ vs. moderate risk $80.0 \%$ vs. high risk $72.9 \%, \mathrm{P}<0.001$; validation cohort five-year DSS: low risk $89.0 \%$ vs. moderate risk $84.3 \%$ vs. high risk $72.2 \%, \mathrm{P}<0.001)$. In the prognostic factor analyses by the Cox proportional hazards regression model in the whole cohort, we confirmed that the LN metastasis risk was also an independent prognostic factor for EGC patients who were over 60 years old (high-risk group: $\mathrm{HR}=1.752,95 \%$ CI: 1.337-2.296; moderate-risk group: $\mathrm{HR}=1.400,95 \% \mathrm{CI}: 1.062-$ 1.848), along with sex, age, $\mathrm{pN}$ stage, and the number of examined LNs (Table 4, P < 0.001). Furthermore, in the relationship analysis, patients in higher-risk groups were confirmed to have a more severe $\mathrm{pN}$ stage (Figure 5C, $\mathrm{P}<0.001$ ).

Table 3. Univariate and multivariate analyses basing on the logistic regression model in the training cohort.

\begin{tabular}{|c|c|c|c|c|c|c|c|}
\hline \multirow[t]{2}{*}{ Characteristics } & & \multicolumn{3}{|c|}{ Univariate analysis } & \multicolumn{3}{|c|}{ Multivariate analysis } \\
\hline & & RR & $95 \% \mathrm{CI}$ & $P$ value & RR & $95 \% \mathrm{CI}$ & $P$ value \\
\hline \multirow[t]{2}{*}{ Sex } & Male & Ref & & 0.760 & & & \\
\hline & Female & 0.957 & $0.723-1.268$ & & & & \\
\hline Age & (continuous) & 0.985 & $0.966-1.005$ & 0.145 & & & \\
\hline \multirow[t]{5}{*}{ Size } & $\leq 1 \mathrm{~cm}$ & Ref & & $<0.001$ & Ref & & $<0.001$ \\
\hline & $>1 \mathrm{~cm}$ and $\leq 2 \mathrm{~cm}$ & 2.224 & $1.303-3.798$ & & 1.697 & $0.981-2.937$ & \\
\hline & $>2 \mathrm{~cm}$ and $\leq 3 \mathrm{~cm}$ & 3.468 & 2.013-5.974 & & 2.492 & $1.425-4.359$ & \\
\hline & $>3 \mathrm{~cm}$ and $\leq 4 \mathrm{~cm}$ & 5.555 & $3.136-9.839$ & & 4.121 & $2.290-7.415$ & \\
\hline & $>4 \mathrm{~cm}$ & 6.447 & $3.756-11.068$ & & 4.607 & $2.644-8.026$ & \\
\hline \multirow[t]{4}{*}{ Grade } & Well differentiated & Ref & & 0.003 & Ref & & 0.014 \\
\hline & Moderately differentiated & 1.729 & $1.066-2.802$ & & 1.624 & $0.981-2.689$ & \\
\hline & Poorly differentiated & 2.197 & $1.388-3.477$ & & 2.009 & $1.240-3.255$ & \\
\hline & Undifferentiated & 3.456 & $1.419-8.418$ & & 3.346 & $1.300-8.608$ & \\
\hline \multirow[t]{4}{*}{ Tumor location } & Upper & Ref & & 0.493 & & & \\
\hline & Middle & 1.553 & $0.613-3.935$ & & & & \\
\hline & Lower & 1.656 & $0.685-4.004$ & & & & \\
\hline & Mixed & 1.361 & $0.555-3.336$ & & & & \\
\hline \multirow[t]{2}{*}{ T stage } & $\mathrm{T} 1 \mathrm{a}$ & Ref & & $<0.001$ & Ref & & $<0.001$ \\
\hline & T1b & 4.245 & 2.919-6.174 & & 3.419 & $2.329-5.019$ & \\
\hline
\end{tabular}

$\mathrm{RR}$, risk ratio; $\mathrm{CI}$, confidence interval. 
Points

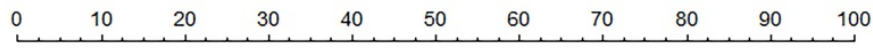

Grade

Well differentiated $\quad$ Moderately differentiated

Size

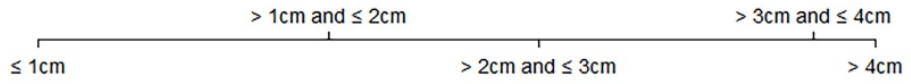

Tstage

T1b

T1a

Total Points

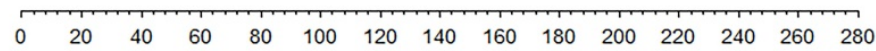

Risk of LN metastasis

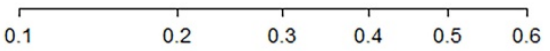

Figure 3. Nomogram predicting lymph node metastasis risk of elderly EGC patients basing on the univariate and multivariate logistic regression models.

(A)

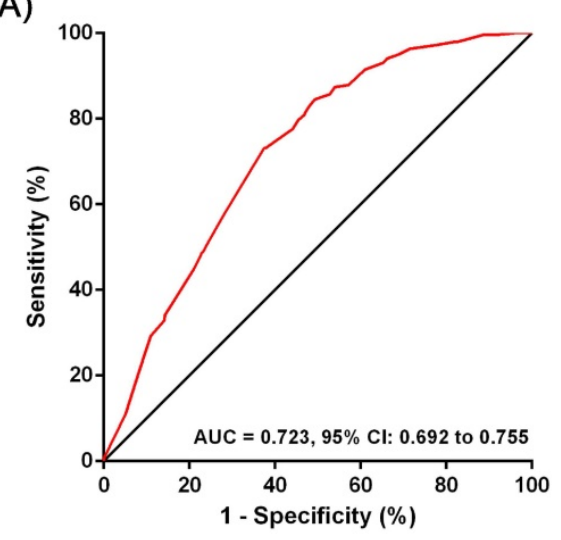

(C)

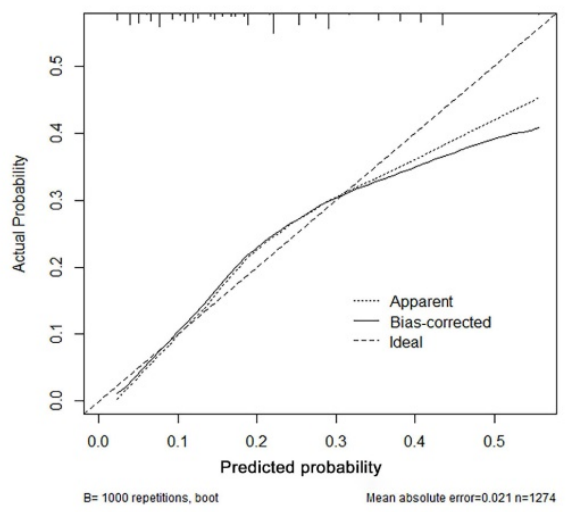

(E)

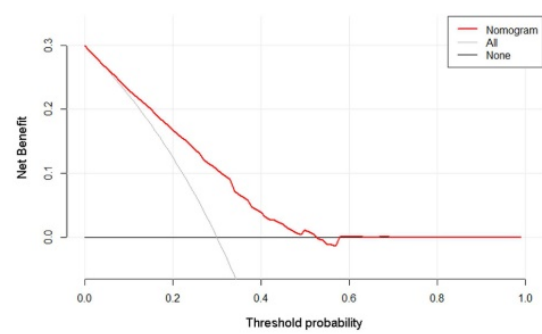

(B)

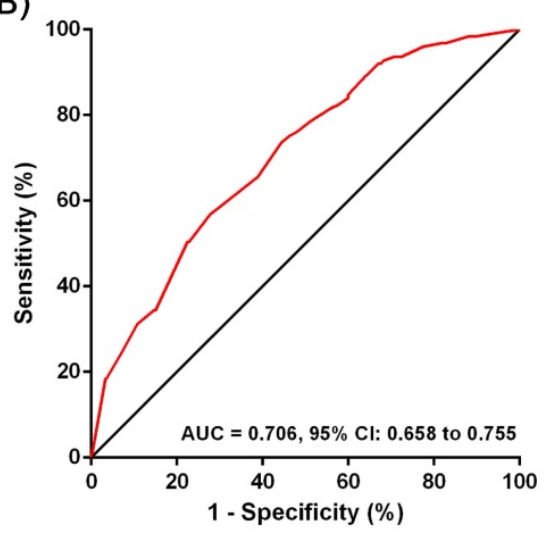

(D)

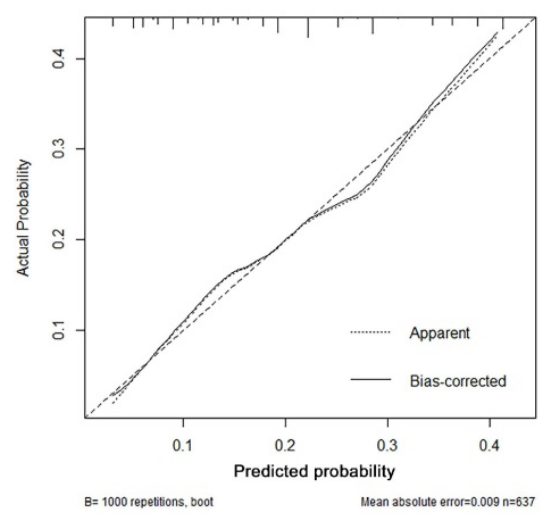

(F)

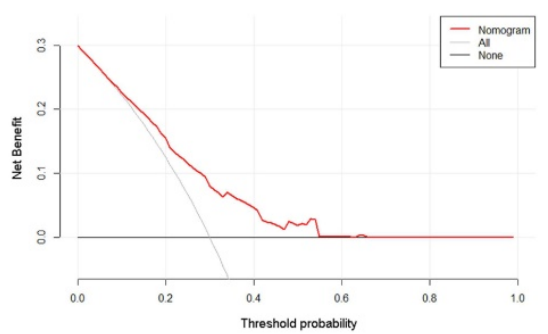

Figure 4. The evaluation of the performance of the nomogram predicting lymph node metastasis in the training cohort and the validation cohort: (A) ROC plot of the nomogram in the training cohort. The AUC was 0.723 (95\% Cl: 0.692-0.755); (B) ROC plot of the nomogram in the validation cohort. The AUC was 0.706 (95\% Cl: $0.658-0.755)$; (C) Calibration plot in the training cohort (1000 repetitions), mean absolute error $=0.021$; (D) Calibration plot in the validation cohort (1000 repetitions), mean absolute error $=$ 0.009 ; (E) The DCA curve of the training cohort; (F) the DCA curve of the validation cohort. 
(A)

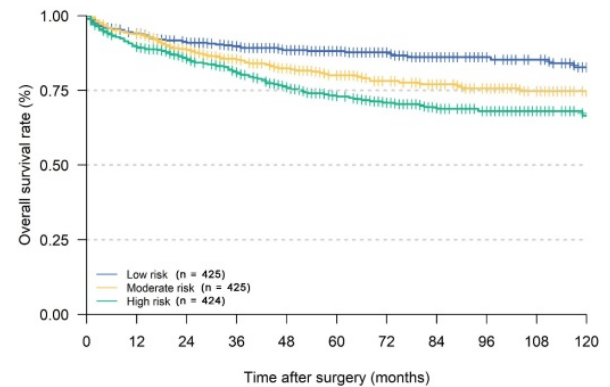

(B)

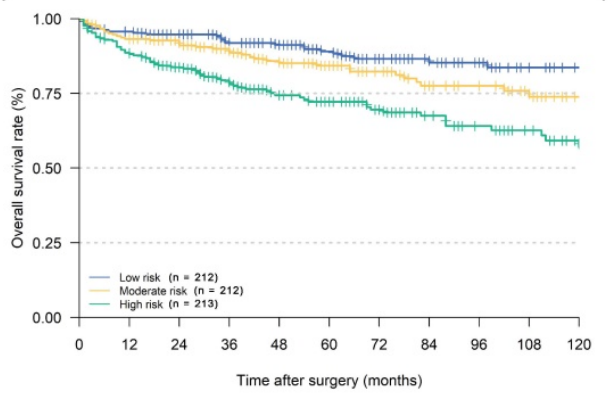

(C)

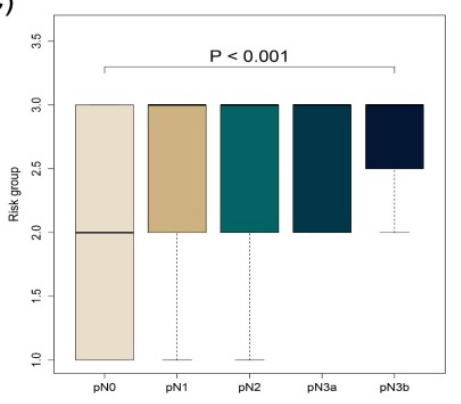

Figure 5. Kaplan-Meier disease-specific survival curves of elderly EGC patients under different degrees of lymph node metastasis risk and the relationship analysis between $\mathrm{pN}$ stage and lymph node metastasis risk: (A) Kaplan-Meier curve of training cohort; (B) Kaplan-Meier curve of validation cohort; (C) relationship between pN stage and lymph node metastasis risk under the Kruskal-Wallis test $(\mathrm{P}<0.001)$.

Table 4. Univariate and multivariate analyses basing on the Cox proportional hazards regression model in the whole cohort.

\begin{tabular}{|c|c|c|c|c|c|c|c|}
\hline \multirow[t]{2}{*}{ Characteristics } & & \multicolumn{3}{|c|}{ Univariate analysis } & \multicolumn{3}{|c|}{ Multivariate analysis } \\
\hline & & HR & $95 \% \mathrm{CI}$ & $\begin{array}{l}P \\
\text { value }\end{array}$ & HR & $95 \% \mathrm{CI}$ & $\begin{array}{l}P \\
\text { value }\end{array}$ \\
\hline \multirow[t]{2}{*}{ Sex } & Male & Ref & & 0.014 & Ref & & 0.002 \\
\hline & Female & 0.774 & $0.631-0.950$ & & 0.722 & $0.587-0.889$ & \\
\hline Age & (continuous) & 1.058 & $1.042-1.073$ & $\begin{array}{l}< \\
0.001\end{array}$ & 1.060 & $1.044-1.075$ & $\begin{array}{l}< \\
0.001\end{array}$ \\
\hline \multirow{4}{*}{$\begin{array}{l}\text { Tumor } \\
\text { location }\end{array}$} & Upper & Ref & & 0.076 & & & \\
\hline & Middle & 0.579 & $0.355-0.942$ & & & & \\
\hline & Lower & 0.554 & $0.356-0.863$ & & & & \\
\hline & Mixed & 0.578 & $0.368-0.909$ & & & & \\
\hline \multirow[t]{5}{*}{ N stage } & No & Ref & & $\begin{array}{l}< \\
0.001\end{array}$ & Ref & & $\begin{array}{l}< \\
0.001\end{array}$ \\
\hline & N1 & 2.036 & $1.562-2.652$ & & 1.903 & $1.420-2.551$ & \\
\hline & N2 & 2.756 & $1.994-3.808$ & & 2.925 & $2.024-4.228$ & \\
\hline & N3a & 3.313 & $1.897-5.787$ & & 3.836 & $2.106-6.987$ & \\
\hline & N3b & 5.297 & $2.184-12.847$ & & 6.731 & 2.673-16.949 & \\
\hline \multirow[t]{2}{*}{ Examined LNs } & $\leq 15$ & Ref & & 0.018 & Ref & & 0.004 \\
\hline & $>15$ & 0.780 & $0.634-0.959$ & & 0.725 & $0.584-0.900$ & \\
\hline \multirow[t]{2}{*}{$\begin{array}{l}\text { Adjuvant } \\
\text { therapy }\end{array}$} & $\begin{array}{l}\text { No/ } \\
\text { unknown }\end{array}$ & Ref & & 0.004 & Ref & & 0.532 \\
\hline & Yes & 0.677 & $0.520-0.881$ & & 1.108 & $0.804-1.527$ & \\
\hline \multirow[t]{3}{*}{$\begin{array}{l}\text { LN metastasis } \\
\text { risk }\end{array}$} & Low & Ref & & $\begin{array}{l}< \\
0.001\end{array}$ & Ref & & $\begin{array}{l}< \\
0.001\end{array}$ \\
\hline & Moderate & 1.533 & $1.166-2.017$ & & 1.400 & $1.062-1.848$ & \\
\hline & High & 2.283 & $1.766-2.952$ & & 1.752 & $1.337-2.296$ & \\
\hline
\end{tabular}

$\mathrm{HR}$, hazard ratio; $\mathrm{CI}$, confidence interval; LNs, lymph nodes.

\section{Discussion}

In the current multicenter study, a prediction nomogram model of LN metastasis - which allowed for the rapid assessment of metastasis risk through preoperative examinations - was established based on a large cohort of elderly patients with EGC. Through our study, we confirmed that the LN metastasis risk assessed by the nomogram was an independent prognostic factor for the current cohort. We thought that the elderly population with EGC under a high or moderate risk of LN metastasis could consider undergoing gastrectomy instead of ESD at the time of initial diagnosis, thus avoiding the trauma caused by a second surgery for some patients.

Nowadays, EMR and ESD have gradually become the optimal treatment choice for EGC patients
[3]. However, these two methods cannot achieve curative resection for every case on account of the limitations of endoscopic and imageological predictions for LN metastasis [5-7]. It was reported that in the Japanese population, LN metastasis was found in $8.4-12.3 \%$ of patients who underwent radical surgery after ESD for EGC $[9,25]$. In the current study, 539 EGC patients from the whole CMU and SEER cohorts before screening for age were diagnosed with LN metastasis, and 371 (68.8\%) were elderly patients. Moreover, in EGC with LN metastasis, recurrence was also inevitable. Approximately $2.2 \%-$ $7.0 \%$ of patients with EGC experienced recurrence after gastrectomy [2, 11, 26, 27]. In addition, T1 patients with a higher $\mathrm{N}$ stage, such as $\mathrm{N} 2$ and N3, had a significantly higher recurrence rate (T1N2: 16.4\%; T1N3a: 38.5\%; T1N3b: 85.7\%) [28]. In the Western world, although ESD was less widely accepted in its early years due to factors such as technical proficiency, recent studies on ESD in Western centers have shown comparable results to that in Eastern [29-31]. In a Western prospective study of 179 EGC underwent ESD, en bloc and R0 resection rates were up to $98.4 \%$ and $90.2 \%$, respectively. The bleeding rate was $6 \%$ and the perforation rate was $1 \%$ [30]. In comparison, multicenter studies of ESD for EGC at Eastern institutions have reported similar en bloc resection rates of $92.7-96.1 \%$ and R0 resection rates of $82.6-94.5 \%$ with bleeding rates of $0.6-9.9 \%$ and perforation rates of $3.6-4.7 \%$ [31]. In a retrospective case-control study including 260 western EGC patients, ESD could significantly reduce the operation time and postoperative hospitalization stay, and prolong 5-year disease-free survival (DFS) (All $\mathrm{P}<0.05$ ) [32]. However, a retrospective analysis based on the National Cancer Database indicated that endoscopic resection was inferior in terms of prognosis for cT1aN0 and cT1bN0 GC when compared with gastrectomy and suggested that a construction of a subset considering the risk of LN metastasis was beneficial to prolong EGC patients' 
survival from endoscopic resection [33]. With the globalization of ESD and the increasing mastery of ESD technology by doctors in the Western world, the application of ESD in the Western world will gradually become widespread. Therefore, it is also of great significance in the Western world to first assess the risk of LNM in elderly patients with EGC and then select the best treatment to reduce the physical trauma caused by unnecessary secondary surgery.

Therefore, there have been studies aimed at establishing scoring systems to assess the risk of $\mathrm{LN}$ metastasis for EGC patients [9, 10, 13]. To evaluate whether reoperation would be necessary after ESD, Hatta et al. [13] established a scoring system known as the eCura system to assess the postoperative LN metastasis rate in 15,785 EGC patients with ESD. The system was adopted by the fifth edition of the Japanese Gastric Cancer Treatment Guideline [14]. The system included tumor size, tumor depth, lymphatic invasion, venous invasion, and vertical margin. It reported that patients with a low risk had a $2.5 \%$ rate of $\mathrm{LN}$ metastasis, intermediate risk a $6.7 \%$ rate of LN metastasis, and high risk a $22.7 \%$ rate of $\mathrm{LN}$ metastasis. Furthermore, the five-year DSS differed significantly among the three groups (low risk: $99.6 \%$; intermediate risk: 96\%; high risk: $90.1 \%)$. The study showed that patients with a low risk were more suitable to undergo ESD without additional treatment. Moreover, the eCura system was a seven-point system. Another 11-point risk-scoring model for LN metastasis was based on 3483 EGC patients and also included five variables: tumor size, tumor depth, histological type, ulcerative findings, and status of lymphovascular invasion [6]. Compared to the 11-point system, the advantages of the eCura system were that it was based on multi-center populations and could evaluate LN metastasis and DSS very well. Mu et al. [10] established a nomogram for 872 EGC patients to predict LN metastasis by four variables: lymphovascular invasion, degree of differentiation, tumor size, and extent of invasion. The nomogram was also designed to determine whether additional LN resection was necessary.

The eCura system of others might be suitable for patients who could successfully tolerate a second surgery, but for some elderly or sickly patients, reoperation might put these patients at risk of death not from the EGC itself. It was also reported that there was a higher risk of complications or death from reoperation [34]. It was also reported that in multivariate analyses, patients over 50 years with $\mathrm{T} 1 \mathrm{~b}$ GC had a significantly higher possibility of LN metastasis than younger patients $(\mathrm{OR}=2.703,95 \% \mathrm{CI}$ : 1.126-6.494, $\mathrm{P}=0.026$ ) [35]. Therefore, it is necessary to establish an assessment system that can differentiate, at the time of initial diagnosis, elderly patients for whom it would be better to undergo radical surgery than ESD.

At present, the diagnostic methods of EGC rely mainly on endoscopic and pathological biopsy. The size and grade of the tumor can be obtained. To determine the depth of invasion, endoscopic ultrasonography has been confirmed to have high accuracy [36]. Thus, according to the former methods, after the initial diagnosis of EGC, the nomogram established in the current study according to the grade, tumor size, and $\mathrm{T}$ stage performed well and could rapidly predict the risk of LN metastasis for elderly patients. It is worth mentioning that the grade obtained by local biopsy is not representative of the whole tumor. Therefore, during endoscopic examination, a multi-point biopsy should be conducted on the tumor site when possible. This would improve the accuracy of the grade. Based on the model, the predicted rate of LN metastasis ranged from $2.3 \%$ to $55.7 \%$ in the whole cohort. In the low-risk, moderate-risk, and high-risk groups, the median rates of $\mathrm{LN}$ metastasis were $6.2 \%, 18.4 \%$, and $35.3 \%$, respectively. Whether to perform ESD or gastrectomy varies in different patients' perceptions, countries, and policies $[14,37,38]$. The current study's risk-prediction system could become a reference for clinical decision making after the initial diagnosis of EGC in elderly patients worldwide.

The current study also has several limitations. First, our analyses were based on retrospective data, and the selection principle was based on diagnosis, demographic and pathological characteristics, and other information existing in the CMU and SEER databases. Second, the molecular factors related to tumor malignancy or LN metastasis were not included in the nomogram. Third, the analyses of adjuvant therapy or neoadjuvant therapy are limited due to the lack of detailed chemotherapy-related information in the both databases. If a favorable response is observed and the tumor regresses to ypT1 from neoadjuvant therapy, it is still not considered to be EGC and these patients are not suitable for endoscopic resection. Although this part of patients was not excluded out in the current study, these patients tended to have larger tumor size and lower degree of differentiation than patients initially diagnosed with EGC, thus having a higher probability of being classified as high-risk patients in our model. Since the prognosis of EGC patients is generally optimistic and the recurrence rate is low, applying DFS to analyze clinical data and constructing the nomogram might be more consistent with the actual clinical situations. However, there is no specific information on DFS in the SEER database. For small 
tumors that are difficult to biopsy at many sites, the representativeness of the diagnostic results of grade would be limited. In this situation, the clinicians' comprehensive and empirical judgment is more important to determine the final treatment of patients. Furthermore, even though we built the training cohort and the validation cohort to evaluate the effectiveness of our model, the external data must still be validated. We will conduct a randomized prospective study including patients receiving neoadjuvant therapy to explore the effectiveness of the model and optimize it.

In conclusion, the nomogram established in the present study could predict the risk of LN metastasis of elderly patients with EGC before ESD or EMR. It is important to minimize the likelihood of reoperation, especially in the elderly population. The value of this nomogram for elderly EGC patients from other institutions will be determined in future studies.

\section{Abbreviations}

EGC: early gastric cancer; LN: lymph node; GC: gastric cancer; ESD: endoscopic submucosal dissection; EMR: endoscopic mucosal resection; mLNs: metastasis lymph nodes; JGCA: Japanese Gastric Cancer Association; CMU: China Medical University; SEER: Surveillance, Epidemiology, and End Results; TNM: tumor, node, and metastasis; AJCC: American Joint Committee on Cancer; ROC: receiver operating characteristic; AUC: area under the curve; DCA: decision curve analysis; DSS: disease-specific survival; OR: odds ratio; CI: confidence interval; DFS: disease-free survival.

\section{Acknowledgements}

The authors appreciate the help and discussion of all members of the Department of Oncology Surgery.

\section{Availability of Data and Materials}

The datasets used and/or analyzed during the current study are available from the corresponding author on reasonable request.

\section{Ethical Statement}

The authors are accountable for all aspects of the work and ensuring that questions related to the accuracy or integrity of any part of the work are appropriately investigated and resolved.

\section{Funding}

This study was supported by grants from the National Natural Science Foundation of China (Grant Nos. 81772549).

(I) Conception and design: HMX, SWP

(II) Administrative support: HMX
(III) Provision of study materials or patients: SWP, WA

(IV) Collection and assembly of data: SWP, YET

(V) Data analysis and interpretation: QCC, PFL

(VI) Manuscript writing: All authors.

(VII) Final approval of manuscript: All authors.

\section{Competing Interests}

The authors have declared that no competing interest exists.

\section{References}

1. Sano T, Coit DG, Kim HH et al. Proposal of a new stage grouping of gastric cancer for TNM classification: International Gastric Cancer Association staging project. Gastric Cancer 2017; 20: 217-225.

2. Lai JF, Kim S, Kim K et al. Prediction of recurrence of early gastric cancer after curative resection. Ann Surg Oncol 2009; 16: 1896-1902.

3. Ono $\mathrm{H}$, Yao K, Fujishiro $\mathrm{M}$ et al. Guidelines for endoscopic submucosal dissection and endoscopic mucosal resection for early gastric cancer. Dig Endosc 2016; 28: 3-15.

4. Liu Q, Ding L, Qiu X, Meng F. Updated evaluation of endoscopic submucosal dissection versus surgery for early gastric cancer: A systematic review and meta-analysis. Int J Surg 2020; 73: 28-41.

5. Ryu KW, Choi IJ, Doh YW et al. Surgical indication for non-curative endoscopic resection in early gastric cancer. Ann Surg Oncol 2007; 14: 3428-3434.

6. Oda I, Gotoda $\mathrm{T}$, Sasako $\mathrm{M}$ et al. Treatment strategy after non-curative endoscopic resection of early gastric cancer. Br J Surg 2008; 95: 1495-1500.

7. Kim ER, Lee $\mathrm{H}$, Min $\mathrm{BH}$ et al. Effect of rescue surgery after non-curative endoscopic resection of early gastric cancer. Br J Surg 2015; 102: 1394-1401.

8. Gotoda T, Yanagisawa A, Sasako M et al. Incidence of lymph node metastasis from early gastric cancer: estimation with a large number of cases at two large centers. Gastric Cancer 2000; 3: 219-225.

9. Sekiguchi $\mathrm{M}$, Oda I, Taniguchi $\mathrm{H}$ et al. Risk stratification and predictive risk-scoring model for lymph node metastasis in early gastric cancer. J Gastroenterol 2016; 51: 961-970.

10. Mu J, Jia Z, Yao $\mathrm{W}$ et al. Predicting lymph node metastasis in early gastric cancer patients: development and validation of a model. Future Oncol 2019; 15: 3609-3617.

11. Youn HG, An JY, Choi MG et al. Recurrence after curative resection of early gastric cancer. Ann Surg Oncol 2010; 17: 448-454.

12. Pan $\mathrm{S}$, Wang $\mathrm{P}$, Xing $\mathrm{Y}$ et al. Retrieved lymph nodes from different anatomic groups in gastric cancer: a proposed optimal number, comparison with other nodal classification strategies and its impact on prognosis. Cancer Commun (Lond) 2019; 39: 49.

13. Hatta W, Gotoda T, Oyama T et al. A Scoring System to Stratify Curability after Endoscopic Submucosal Dissection for Early Gastric Cancer: "eCura system". Am J Gastroenterol 2017; 112: 874-881.

14. Japanese Gastric Cancer Treatment Guideline 2018 (ver.5). (http://www.jgca.jp/guideline/fifth/index.html).

15. Wang J, Dang P, Raut CP et al. Comparison of a lymph node ratio-based staging system with the 7th AJCC system for gastric cancer: analysis of 18,043 patients from the SEER database. Ann Surg 2012; 255: 478-485.

16. Son T, Sun J, Choi S et al. Multi-institutional validation of the 8th AJCC TNM staging system for gastric cancer: Analysis of survival data from high-volume Eastern centers and the SEER database. J Surg Oncol 2019; 120: 676-684.

17. In H, Solsky I, Palis B et al. Validation of the 8th Edition of the AJCC TNM Staging System for Gastric Cancer using the National Cancer Database. Ann Surg Oncol 2017; 24: 3683-3691.

18. Balachandran VP, Gonen M, Smith JJ, et al. Nomograms in oncology: more than meets the eye. Lancet Oncol. 2015; 16(4):e173-80

19. Moons KG, Kengne AP, Woodward $M$ et al. Risk prediction models: I. Development, internal validation, and assessing the incremental value of a new (bio)marker. Heart 2012; 98: 683-690.

20. Hanley JA, McNeil BJ. The meaning and use of the area under a receiver operating characteristic (ROC) curve. Radiology 1982; 143: 29-36.

21. Steyerberg EW, Harrell FE, Jr., Borsboom GJ et al. Internal validation of predictive models: efficiency of some procedures for logistic regression analysis. J Clin Epidemiol 2001; 54: 774-781.

22. Huang L, Jansen L, Balavarca Y et al. Significance of Examined Lymph Node Number in Accurate Staging and Long-term Survival in Resected Stage I-II Pancreatic Cancer-More is Better? A Large International Population-based Cohort Study. Ann Surg 2019; [Online ahead of print]. doi: 10.1097/SLA.0000000000003558.

23. Schemper M, Smith TL. A note on quantifying follow-up in studies of failure time. Control Clin Trials 1996; 17: 343-346.

24. Altman DG, De Stavola BL, Love SB, Stepniewska KA. Review of survival analyses published in cancer journals. Br J Cancer 1995; 72: 511-518. 
25. Hatta $\mathrm{W}$, Gotoda $\mathrm{T}$, Oyama $\mathrm{T}$ et al. Is radical surgery necessary in all patients who do not meet the curative criteria for endoscopic submucosal dissection in early gastric cancer? A multi-center retrospective study in Japan. J Gastroenterol 2017; 52: 175-184.

26. Cao L, Selby LV, Hu X et al. Risk factors for recurrence in T1-2N0 gastric cancer in the United States and China. J Surg Oncol 2016; 113: 745-749.

27. Lo SS, Wu CW, Chen JH et al. Surgical results of early gastric cancer and proposing a treatment strategy. Ann Surg Oncol 2007; 14: 340-347.

28. Yura M, Yoshikawa T, Otsuki S et al. Is surgery alone sufficient for treating T1 gastric cancer with extensive lymph node metastases? Gastric Cancer 2020; 23 : 349-355.

29. Michael X Ma, Michael J Bourke. Endoscopic submucosal dissection in the West: Current status and future directions. Digestive Endoscopy. 2018; 30(3): 310-320.

30. Probst A, Schneider A, Schaller T, et al. Endoscopic submucosal dissection for early gastric cancer: Are expanded resection criteria safe for Western patients? Endoscopy 2017; 49: 855-65.

31. Choi IJ, Lee NR, Kim SG et al. Short-term outcomes of endoscopic submucosal dissection in patients with early gastric cancer: A prospective multicenter cohort study. Gut. Liv. 2016;10: 739-48.

32. Bausys R, Bausys A, Stanaitis J, et al. Propensity score-matched comparison of short-term and long-term outcomes between endoscopic submucosal dissection and surgery for treatment of early gastric cancer in a Western setting. Surg Endosc. 2019; 33(10):3228-3237.

33. Sivesh K Kamarajah, Sheraz R Markar, Alexander W Phillips, et al. Local Endoscopic Resection is Inferior to Gastrectomy for Early Clinical Stage T1a and T1b Gastric Adenocarcinoma: A Propensity-Matched Study. Ann Surg Oncol. 2021. [Online ahead of print]. doi: 10.1245/s10434-020-09485-4.

34. Sah BK, Chen MM, Yan M, Zhu ZG. Reoperation for early postoperative complications after gastric cancer surgery in a Chinese hospital. World J Gastroenterol 2010; 16: 98-103.

35. Zheng Z, Zhang Y, Zhang $\mathrm{L}$ et al. Nomogram for predicting lymph node metastasis rate of submucosal gastric cancer by analyzing clinicopathological characteristics associated with lymph node metastasis. Chin J Cancer Res 2015; 27: 572-579.

36. Kim J, Kim SG, Chung H et al. Clinical efficacy of endoscopic ultrasonography for decision of treatment strategy of gastric cancer. Surg Endosc 2018; 32: 3789-3797.

37. NCCN Practice Guidelines in Oncology (Gastric Cancer)-V4.2019 (www.nccn.org).

38. Smyth EC, Verheij M, Allum W et al. Gastric cancer: ESMO Clinical Practice Guidelines for diagnosis, treatment and follow-up. Ann Oncol 2016; 27: v38-v49. 